\title{
Displacement Sensitivity Control for Beams and Plates using Electro-Optic Holography
}

\author{
Jonathan D. Blotter \\ Department of Mechanical Engineering, Brigham Young University, Provo, Utah 84602-4201, USA
}

\begin{abstract}
Tony J. Anderson
Department of Mechanical Engineering, University of Idaho, Moscow, Idabo 83844, USA
\end{abstract}

(Received 1 March 2002; accepted 17 April 2003)

\begin{abstract}
Electro-Optic Holography (EOH) is a whole-field, laser-based, displacement measurement technique. In conventional $\mathrm{EOH}$, the displacement sensitivity is fixed by the wavelength of the laser source. When infrared diode lasers are used, the fringe patterns can become highly dense and unresolvable, limiting the measurable range of displacements. This paper presents and extends a technique for controlling the displacement sensitivity of an EOH system using diode laser current modulation. This technique is known as Frequency Translated ElectroOptic Holography (FTEOH). Using diode laser current modulation, the fringe patterns are based on higher order Bessel functions and the sensitivity of the EOH system can either be increased or decreased. The amount of sensitivity reduction is limited only by the frequency limit of the signal generator that modulates the current supplied to the diode laser. EOH and FTEOH experimental results for a cantilever beam and a simply supported plate are presented and shown to agree well.
\end{abstract}

${ }^{\dagger}$ Member of the International Institute of Acoustics and Vibration (IIAV)

\section{INTRODUCTION}

Electro-Optic Holography (EOH) is a laser-based, wholefield, displacement measurement system. ${ }^{1-6}$ A schematic of the EOH system is shown in Fig. 1. To create an EOH interference pattern, a light beam from a monochromatic laser is split into two beams, as shown. The portion of the split beam denoted as the object beam is directed through a microscope objective lens for expansion and then onto the object of interest. The light scattered by the object is frequency modulated by an amount equal to the object excitation frequency plus the integer multiples (higher harmonics). It is important to note that the scattered object beam is also phase modulated from the original form. ${ }^{7}$ The reference beam is unmodulated. A portion of the object beam is captured through a camera lens and combined with the reference beam through a beam splitter. A CCD camera records the interference that develops when these two beams are combined.

Infrared diode lasers are efficient light sources for $\mathrm{EOH}$ systems. The wavelength of infrared diode lasers typically ranges from $800-900 \mathrm{~nm}$. In this research, a diode laser with a wavelength of $852 \mathrm{~nm}$ was used as the light source. For out-of-plane measurements, one complete fringe cycle represents a displacement magnitude approximately equal to the wavelength of the laser. With this wavelength and the relatively large displacement amplitudes present in many practical vibrating structures, the fringe density of an $\mathrm{EOH}$ interference pattern is often so high that it is difficult or impossible to detect and resolve the individual fringes. Therefore, the ability to control the sensitivity or number of fringes that represent the displacement field is vitally important when measuring displacements on vibrating structures.

Figure 2 illustrates a fringe pattern acquired from a plate simply supported on all four sides. The plate is shown to be vibrating near the $2-1$ mode. The zero order fringe (ZOF) is the bright diagonal line and represents a location of zero displacement (node lines). ${ }^{1}$ In Fig. 2, the zero order fringe can be detected but the remaining fringe density is at the onset of being so high that it would be difficult to extract the displacement field. The goal of the FTEOH technique is to take the image shown in Fig. 2 and without changing the amplitude of the displacements, control the fringe pattern so that the individual fringes are easily identified. The basic idea is to increase the amount of displacement that each fringe represents and thereby reduce the number of fringes required to represent the same displacement.

EOH differs from conventional holography in that the EOH images are directly recorded and processed by a CCD camera-based video system which provides a near real-time display of the interference fringes. In conventional, filmbased holography, previous research has shown some fringe control effects by modulating the reference beam using vibrating mirrors. ${ }^{8}$ However, these methods have only shown the fringe reduction and not shown or discussed methods to extract the displacements from the reduced fringe maps. This paper extends the basic idea of laser beam modulation to the CCD camera-based EOH technique and presents a method for extracting the displacements from the reduced fringe maps. The procedure to extract the displacements is presented and FTEOH and EOH displacement results for a cantilever beam are computed and compared. The FTEOH method is also shown to account for 2-D plate images. This extension is shown by comparing FTEOH and EOH results for a plate simply supported on all four sides. Furthermore, instead of using mirrors to obtain the frequency shift in the reference beam, a technique based on diode laser current modulation is presented and used to obtain the fringe control. Diode laser current modulation provides a significant enhancement compared to the mirror-based systems in that the 